\title{
Minocycline fails to modulate cerebrospinal fluid HIV infection or immune activation in chronic untreated HIV-1 infection: results of a pilot study
}

\author{
Emily L Ho ${ }^{1,4}$, Serena S Spudich ${ }^{1,5}$, Evelyn Lee ${ }^{1}$, Dietmar Fuchs ${ }^{2}$, Elizabeth Sinclair ${ }^{3}$ and Richard W Price ${ }^{1 *}$
}

\begin{abstract}
Background: Minocycline is a tetracycline antibiotic that has been shown to attenuate central nervous system (CNS) lentivirus infection, immune activation, and brain injury in model systems. To initiate assessment of minocycline as an adjuvant therapy in human CNS HIV infection, we conducted an open-labelled pilot study of its effects on cerebrospinal fluid (CSF) and blood biomarkers of infection and immune responses in 7 viremic subjects not taking antiretroviral therapy.

Results: There were no discernable effects of minocycline on CSF or blood HIV-1 RNA, or biomarkers of immune activation and inflammation including: CSF and blood neopterin, CSF CCL2, CSF white blood cell count, and expression of cell-surface activation markers on CSF and blood T lymphocytes and monocytes.
\end{abstract}

Conclusions: This pilot study of biological responses to minocycline suggests little potential for its use as adjunctive antiviral or immunomodulating therapy in chronic untreated HIV infection.

\section{Background}

Human immunodeficiency virus type one (HIV) infection of the central nervous system (CNS) is a nearly ubiquitous facet of systemic infection that begins early after exposure [1-6]. This CNS infection is accompanied by local immune responses that are reflected in elevations of CSF biomarkers of immune activation and inflammation [7-11]. Though clinically inapparent in most patients, CNS HIV infection evolves in some to a more 'invasive' HIV encephalitis (HIVE) that manifests with the cognitive and motor dysfunction characteristic of the AIDS dementia complex (ADC) [12], now commonly referred to as HIV-associated dementia (HAD) [13]. While the pathogenesis of brain injury related to HIVE is not precisely understood, it likely involves 'indirect' pathways of injury in which host inflammatory mediators serve as important neuropathogenic signals and toxins and, hence, in a broad sense can be considered immunopathological $[14,15]$. Chronic subclinical CNS infection may also be accompanied by more

\footnotetext{
* Correspondence: rwprice@sfgh.ucsf.edu

'Department of Neurology' University of California San Francisco, San Francisco, CA, USA

Full list of author information is available at the end of the article
}

indolent brain injury that manifests later as cognitive impairment $[13,16,17]$ and possibly continues despite antiretroviral treatment [18]. Although the pathogenesis of this type of chronic injury is less well understood than that of HIVE, continued immune activation may be an important factor $[8,19,20]$.

These indirect mechanisms of injury have led to a search for adjuvant modes of treatment to mitigate brain injury by attenuating immunopathology or interfering with downstream neurotoxic pathways. While a number of adjunctive therapies have been advocated or tested [21], none of these has yet proved effective or entered clinical practice. Recently, the antibiotic, minocycline, has been proposed as a candidate therapy in this broad class. Minocycline has been shown to reduce lentivirus infection and immune responses in model systems [22-27] and also to exert neuroprotective effects in diverse models of neurodegeneration [28-35]. This has led to the suggestion that it might be useful in human HIV infection, either as an adjunct to [25] or low-cost replacement for antiretroviral treatment, with particular relevance to attenuation of CNS infection and disease. To begin to test this in the human disease setting, we initiated a pilot study to evaluate minocycline in chronic
C Biomed Central

() 2011 Ho et al; licensee BioMed Central Ltd. This is an Open Access article distributed under the terms of the Creative Commons Attribution License (http://creativecommons.org/licenses/by/2.0), which permits unrestricted use, distribution, and reproduction in any medium, provided the original work is properly cited. 
human HIV infection in the absence of antiretroviral therapy, using CSF and blood biomarkers as principal indices of drug effects, with CSF infection thus serving as a 'model' of and window into CNS infection and immunoactivation [36,37]. For this open-labelled pilot study we hypothesized that minocycline would reduce CSF HIV-1 RNA concentrations, both absolutely and in relation to blood HIV-1 RNA, and diminish evidence of CSF and blood immune activation, including CSF and blood concentrations of neopterin [11,38], CSF concentrations of CCL2 (monocyte chemotactic protein-1, MCP-1) [39,40] and T cell and monocyte expression of cell-surface activation markers [10].

\section{Results}

Of 17 subjects screened over a period of 3 years (20062009), 6 were excluded because of low CSF HIV-1 RNA $(\mathrm{N}=3)$ or unsuccessful lumbar punctures $(\mathrm{N}=3)$. Three other subjects withdrew from the study without starting minocycline treatment. One subject enrolled in the study but stopped after 4 days due to a reaction to minocycline (nausea and vomiting) that resolved after stopping the drug. The remaining 7 subjects entered the study and were prescribed minocycline. Their baseline characteristics are shown in Table 1. Six of these completed the study without adverse events. One subject discontinued minocycline after week 4 of the study due to elevations in serum transaminases, but continued study participation through the washout period and the last visit at week 14; the transaminases subsequently returned to normal. For repeated measures ANOVA analysis, this subject's 4-week results were carried forward and included in the 8-week data. The six remaining subjects tolerated the treatment without clinical or laboratory evidence of toxicity.

Figure 1 shows the changes from baseline in the primary and secondary outcome measures. There were no significant changes in the virological measures. Both the CSF (A) and plasma (B) HIV-1 RNA remained stable, as did the CSF:plasma HIV-1 RNA ratio (not shown). Likewise, neither the CSF (C) nor plasma (D) neopterin changed. Similarly, none of the CSF or blood T cell (E - H) or monocyte (I and J) activation levels changed. There was no reduction in the CSF WBC count (K), which is composed principally of blood-derived T cells $[10,41,42]$. CSF CCL2 (L), CSF:blood albumin ratio (M), and the brief measure of neurological performance, the QNPZ-4 score $(\mathrm{N})$, also did not change significantly. Curiously, there was a reduction of absolute $\mathrm{CD} 8+(\mathrm{O})$ and $\mathrm{CD} 4+(\mathrm{P})$ $\mathrm{T}$ cell numbers in the blood, although only the latter was statistically significant by repeated measures analysis.

\section{Discussion}

This pilot study was undertaken to explore the use of minocycline as an adjuvant treatment for chronic HIV
Table 1 Baseline subject characteristics

\begin{tabular}{|c|c|c|}
\hline & Median & Range \\
\hline Age (years) & 49.9 & $32.0-55.2$ \\
\hline Gender (M:F) & $6: 1$ & \\
\hline Time since HIV diagnosis (years) & 17.0 & $1.7-20.3$ \\
\hline \multicolumn{3}{|l|}{ HIV-1 RNA ( $\log _{10}$ copies/mL) } \\
\hline Plasma & 4.49 & $4.26-5.56$ \\
\hline CSF & 3.87 & $3.11-4.47$ \\
\hline Plasma:CSF difference & 1.06 & $0.12-1.58$ \\
\hline \multicolumn{3}{|l|}{ Blood T cells (cells/ $/ \mathrm{LL})$} \\
\hline CD4+ & 453 & $267-806$ \\
\hline CD8+ & 1,009 & $575-2185$ \\
\hline 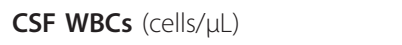 & 9 & $2-18$ \\
\hline \multicolumn{3}{|l|}{ Neopterin (nmol/L) } \\
\hline CSF & 13.1 & $5.9-41.2$ \\
\hline Plasma & 13.4 & $9.2-53.5$ \\
\hline $\operatorname{CSF}$ CCL2 $(\mathrm{pg} / \mathrm{mL})$ & 479.2 & $397.9-1322.2$ \\
\hline \multicolumn{3}{|c|}{ T Cell Activation (percent CD38+/HLA-DR+) } \\
\hline CSF CD4+ & 14.7 & $3.4-60.2$ \\
\hline Blood CD4+ & 13.3 & $7.6-24.7$ \\
\hline CSF CD8+ & 83.4 & $41.8-97.6$ \\
\hline Blood CD8+ & 58.5 & $34.5-78.0$ \\
\hline \multicolumn{3}{|c|}{ Monocyte Activation (percent CD16+) } \\
\hline CSF monocytes & 93.6 & $80.1-100$ \\
\hline Blood monocytes & 10.8 & $4.7-17.0$ \\
\hline CSF:blood albumin ratio & 5.05 & $3.91-12.26$ \\
\hline QNPZ-4 & -0.32 & $-3.44-0.54$ \\
\hline
\end{tabular}

infection, particularly for attenuating the CNS components of immunoactivation and infection. It aimed to provide a preliminary view of the biological effects of minocycline on CNS HIV immune reactions and infection, and to obtain effect-size estimates for power calculations prior to planning a larger controlled trial. Our underlying mechanistic hypotheses centered on the proposed capacity of minocycline to attenuate CNS immune and systemic perturbations and their effects on CNS infection as revealed by changes in CSF and blood biomarkers. We hypothesized that attenuating these immunological effects would be reflected in reductions in CSF (and perhaps plasma) neopterin and CSF CCL2 concentrations, and in the expression of surface activation 


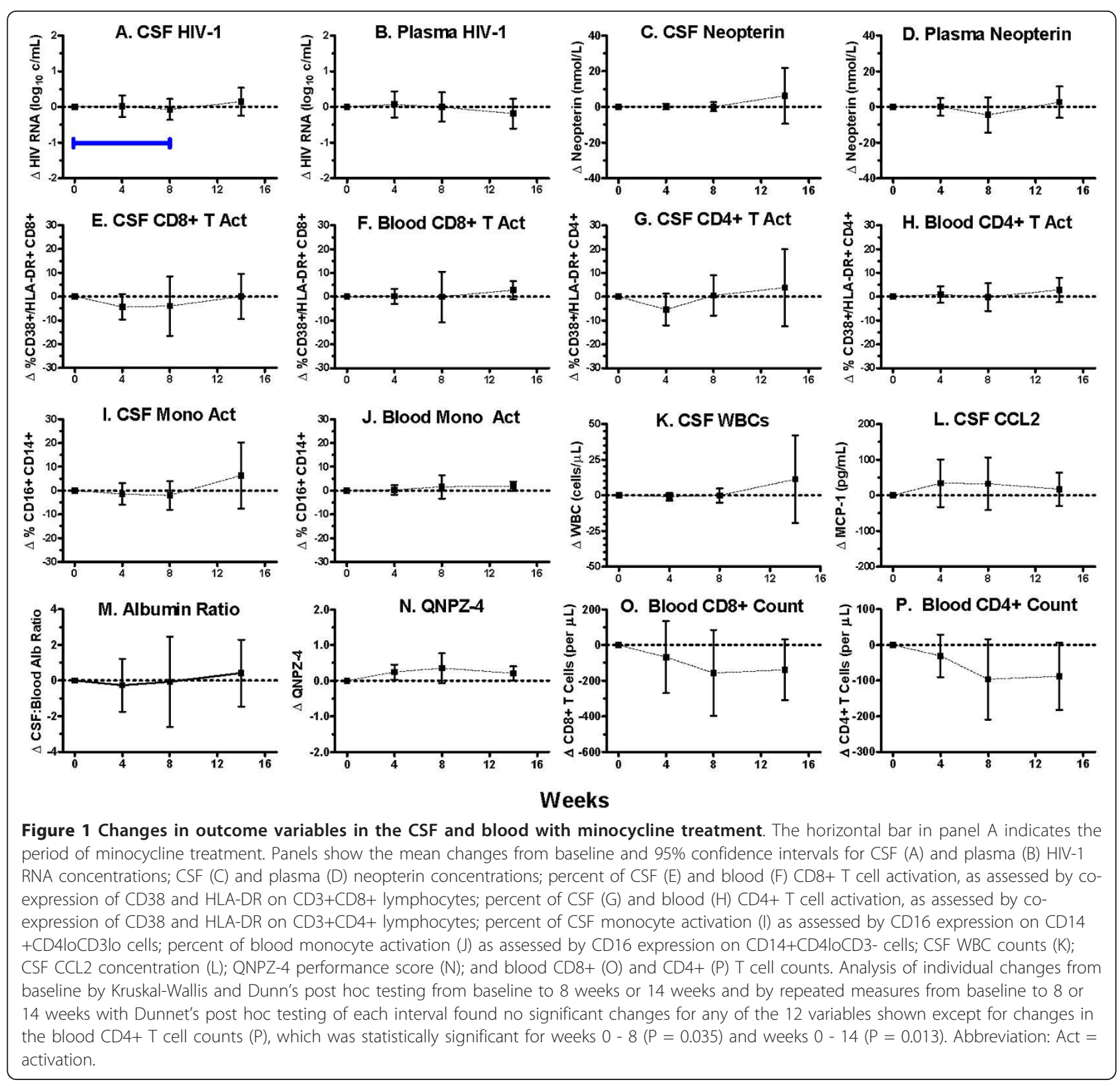

markers on T cells and monocytes. Additionally, we hypothesized that minocycline might also indirectly reduce CNS infection through its effects on various immune system-related mechanisms that contribute to the magnitude of CNS (and CSF) infection, including: $\mathrm{CD} 4+\mathrm{T}$ cell traffic that brings both infected cells and uninfected targets into the CNS, and CD4+ T cell and macrophage activation that enhance viral replication in these cell types. Unfortunately, in this study, none of these effects were seen. Similarly, there were no changes in the other secondary endpoints, including CSF WBC counts, CSF:blood albumin ratios or the brief neurological performance battery, the QNPZ-4.
Minocycline, a licensed tetracycline antibiotic, has been reported to have a number of properties that make it an attractive adjuvant therapy candidate. In various model systems, it has been shown to have anti-inflammatory effects $[43,44]$, including modulation of $\mathrm{T}$ cell activation and attenuation of macrophage and microglial activation $[27,34,45,46]$. It also has neuroprotective properties in vitro and in in vivo animal models [47-52]. These and other properties have led to trials of minocycline in several conditions, including rheumatoid arthritis [53], and neurodegenerative and neuroinflammatory diseases $[33,52,54,55]$. Minocycline also has been shown to inhibit HIV replication in microglia in vitro [22]. 
Importantly, in an SIV model of accelerated CNS infection, minocycline-treated SIV-infected macaques were noted to have less severe encephalitis, reduced expression of CNS inflammatory markers, reduced axonal degeneration and lower levels of CNS virus replication [23]. Recent in vitro studies on human peripheral blood $\mathrm{CD} 4+\mathrm{T}$ cells demonstrate that minocycline has antiviral effects in CD4+ T cells and reduces cellular CD4+ $\mathrm{T}$ cell activation [27]. Since all of these properties made it an intriguing candidate for adjuvant use in CNS HIV infection, our study results thus beg the issue of why we did not see similar effects in the studied patients.

While it is possible that the CSF measurements were insensitive to salutary effects on the brain parenchyma, including the important perivascular environment, this does not seem likely. CSF neopterin is a marker of CNS macrophage activation (presumably including both brain and meningeal populations) that increases with disease severity and is especially elevated in HIVE/HAD $[11,38]$. This pteridine biomarker responds well to antiretroviral therapy [11], although it does not always return to normal levels $[8,19,56]$. Its blood concentration is also a prognostic marker of disease progression [57]. Both CSF and blood levels were unaffected by minocycline in our study, suggesting that there was little effect on CNS or systemic macrophage activation. Similarly, CSF CCL2, a biomarker of macrophage chemotaxis that is also characteristically elevated in HAD/HIVE [58], showed no changes. This is especially disappointing since CSF CCL2 has been used as a biomarker in SIV encephalitis, and was shown to be reduced by minocycline treatment in the SIV model $[23,40]$. Increased levels of CD4+ T cell, CD8 + T cell and monocyte activation observed in the CSF compared to the blood is characteristic of HIV infection $[10,42,59]$ and is likely an important component of both systemic [60-62] and CNS disease pathogenesis $[10,20]$. These measures also were stable through the course of minocyline treatment.

CSF HIV-1 RNA levels reflect more than one cellular source, with the relative contributions differing depending on the stage of systemic and CNS infection and disease evolution [63-66]. Short-lived cells, presumably CD4+ T cells, contribute a CSF viral population that is genetically similar to the blood population [63]. This component has been termed transitory infection $[5,37]$ and is presumably sustained by infected and susceptible CD4+ T cells trafficking into the meninges and brain. In early HIV infection, this type of infection predominates and may even be the only type detected [67]. A second viral population turns over more slowly [66]. This population is likely derived from macrophages, and is genetically distinct from the blood population. This component, termed autonomous or compartmentalized infection, is characteristically detected as a minor contributor to CSF HIV levels in neuroasymptomatic chronic infection, but predominates in more advanced infection, particularly HIV encephalitis (HIVE) [64].

Minocycline might attenuate both types of infection by its effects on $\mathrm{T}$ cell and monocyte-macrophage activation. In the case of transitory infection, $T$ cell activation is critical to support HIV replication and also promotes $\mathrm{T}$ cell traffic that carries infected and uninfected target $\mathrm{CD} 4+\mathrm{T}$ cells into the meninges and perivascular spaces. Hence, if minocycline alters these $\mathrm{T}$ cell properties it might reduce this type of CSF infection. Similarly, activation is likely important for macrophages in sustaining infection and also, perhaps, in their entry into the CNS, including into the perivascular spaces, meninges and parenchyma. Minocycline might, therefore, reduce this type of autonomous infection. However, we detected no evidence of reduced CSF infection, although in the subjects studied with relatively preserved blood CD4+ T cell counts, the major CSF viral population likely originated from transitory type infection, although this was not directly examined in these subjects.

Our methods of examining the hypothesized actions of minocycline should have been adequate to detect a substantial immunological or virological effect of minocycline. Possible reasons as to why there were no discernable effects similar to those in the SIV-infected pigtailed macaques may have included species differences. Perhaps more likely were differences in the disease targets. The SIV model differs from our subjects in the relatively short disease duration and the presence of frank lentivirus encephalitis [23]. Our study patients had a chronic 'stable' infection for a number of years and thus, perhaps, presented a level of immune activation and viral replication that the drug effect was too weak to modify. In addition, the absence of encephalitis meant that there might have been little CNS disease to target. Our study, of course, did not address these possibilities.

The study also did not assess the more direct neuroprotective properties of minocycline. With one exception, our subjects were largely neuroasymptomatic, and we performed only brief quantitative neurological performance testing (QNPZ-4) on four measures. The small improvement noted in this measure, which was not statistically significant, might have related to practice effect. However, if the observed improvement was indeed real, then a study with 20-25 subjects in each of two treatment arms (minocycline and placebo) would be needed for an $80 \%$ power to detect the difference found here at 8 weeks. An AIDS Clinical Trials Group study is studying whether minocycline might improve performance in cognitively impaired HIV-infected subjects (http://clinicaltrials.gov/ct2/show/NCT00361257), and these issues should be addressed by that study. 
The observed decline in blood CD4+ and CD8+ T cell counts was unexpected and unexplained. Curiously, it did not impact the CSF WBC count. This mild T-cell lymphopenia needs to be verified in a larger study, and if so, subject to further investigation.

Overall, this pilot study was subject to several inherent design limitations, including its small size, relatively short duration, and absence of an untreated control group for comparison, raising concern for Type II error. Thus, we cannot fully dismiss the possibility that the study was underpowered to detect a mild effect of the drug or that CSF HIV and CNS immune activation might decline further with longer exposure. However, given the minimal changes noted in the major outcomes, it would take a large study to test the effectiveness of minocycline on these measures in this type of patient population. For example, if the small reduction (-0.070 $\log 10$ copies/mL) in CSF HIV-1 RNA at 8 weeks was indeed a 'real' finding, then it would require more than 100 subjects in each of the two arms (minocycline and placebo) to have an $80 \%$ power to detect this difference between the groups, a difference with likely little clinical meaning. In the case of CSF neopterin, there was no statistically significant reduction, but if the slight increase at 8 weeks $(0.033 \mathrm{nmol} / \mathrm{L})$ was inverted and actually a reduction, it would take 500 subject in each group to detect this difference. Thus, the effects of minocycline on infection and immune activation appeared too weak to justify a study of the requisite size, particularly when viewed in comparison to the potent effects of combination antiretroviral on these variables [6].

\section{Conclusions}

In conclusion, this small pilot study suggests that any effects of minocycline on CNS HIV infection and immune activation were not sufficient to impact chronic HIV in the absence of antiretroviral treatment. Therefore, there seems little justification or indeed ethical basis for treating chronic HIV infection with minocycline instead of combination antiretroviral drugs. However, given the reported in vitro and SIV effects of this tetracycline [23], there still may be reason for further study, for example in well-treated patients in which the level of immunoactivation is partially attenuated or in patients with cognitive impairment in which its neuroprotective properties may yet prove useful in concert with combination antiretroviral treatment.

\section{Methods}

This study was approved by the University of California San Francisco Committee on Human Research and conducted according to the principles expressed in the Declaration of Helsinki. Informed written consent was obtained from all subjects. The study was registered with ClinicalTrials.gov (number: NCT01064752).

\section{Study design}

This was an open-labelled, uncontrolled, pilot study examining the effects of $100 \mathrm{mg}$ of minocycline taken orally twice daily for 8 weeks. Subject entry criteria included: $\geq 18$ years of age; chronic HIV infection with plasma and CSF HIV-1 RNA concentrations $>1,000$ copies/mL; not taking antiretroviral therapy (either naïve to therapy or $>6$ weeks off treatment with no plans to start during the period of study); predicted medication adherence; blood CD $4+\mathrm{T}$ cell counts $>100$ cells $/ \mu \mathrm{l}$; no previous adverse reaction to tetracyclines; no tetracycline treatment for the past 6 months; no contraindications to lumbar puncture (LP); no active opportunistic infection or neurological disease confounding evaluations; ADC stage $<1$ [68]; no concomitant medications altering the metabolism or risk of minocycline; hemoglobin $>10 \mathrm{~g} / \mathrm{dL}$ and liver transaminases $<2.5$ times upper limit of normal; and not taking any other immunomodulating drugs. After consent, subjects underwent a screening evaluation that included lumbar puncture (LP) and CSF characterization, concurrent blood sampling, and standardized neurological assessments as previously described $[6,10,69]$. For those meeting entry criteria, this also served as the baseline visit, and they starting minocycline $100 \mathrm{mg}$ twice daily orally for the next 8 weeks. At four and eight weeks, and after a 6-week washout period off minocycline, subjects underwent repeated evaluation similar to the baseline, including LP and CSF analysis $[6,10,69]$. Treatment adherence was assessed at each onstudy visit by direct questioning and pill count.

The primary outcome measures were the change from baseline during treatment in CSF HIV-1 RNA and CSF neopterin concentrations as indices of CNS infection and immunoactivation [38]. Change from baseline was calculated at weeks four and eight after initiation of minocycline treatment and after a 6-week wash-out period. Additional secondary measured outcomes included changes in: CSF white blood cell (WBC) count; blood CD4+ and CD8+ counts; ratio of CSF to blood albumin as a measure of blood-brain barrier permeability [70,71]; CSF CCL2 as a measure of monocyte-macrophage chemotaxis [58]; CSF and blood CD4+ and CD8+ T cell and monocyte activation as measured by multiparameter flow cytometry [10]. Four quantitative tests (timed gait, grooved pegboard, finger tapping and digit symbol) were used to obtain a simple quantitative neurological performance aggregate score (QNPZ-4) [72].

\section{CSF and blood assays}

HIV-1 RNA was measured in cell-free CSF and plasma by the Roche Amplicor HIV-1 Monitor assay (versions 
1.0 and 1.5, Roche Diagnostic Systems, Inc., Branchburg, N.J). Neopterin concentrations in cell-free CSF and plasma were measured in batch by ELISA according to the manufacturer's instructions (BRAHMS Aktiengesellschaft, Hennigsdorf, Germany). Blood CD4+ and $\mathrm{CD} 8+\mathrm{T}$ cell counts were performed in the San Francisco General Hospital (SFGH) Clinical Laboratories using standard flow cytometric methods. CCL2 was measured in cell-free CSF by ELISA (R\&D Systems, Minneapolis, MN). Other measurements performed in the SFGH Clinical Laboratories using routine clinical methods included CSF and blood albumin (used to compute the CSF:blood albumin ratio $[70,71])$, CSF WBC counts and differential, CSF total protein and blood metabolic profile.

CSF and blood CD4+ and CD8+ T cell activation were assessed by the percent of these cells in fresh specimens co-expressing surface CD38 and HLA-DR by multiparameter flow cytometry as previously described [10]. Blood monocytes were defined as CD14+CD4loCD3cells from the mononuclear gate. CSF monocytes had low level staining for CD3 and were defined as CD14 + CD4loCD3lo cells. Monocyte activation was defined by the percent of these cells expressing CD16 [10]. Flow cytometry data was compensated and analysed with FlowJo (Tree Star, Ashland, OR).

\section{Statistics}

Changes from baseline to follow-up test intervals were analysed by Kruskal-Wallis test with Dunn's post hoc comparison of individual intervals and additionally from baseline through week 8 using repeated measures ANOVA with Dunnet's post hoc comparison. All P values were two-sided with values $<0.05$ considered significant. Statistical analyses used Prism 5 (GraphPad Software Inc, San Diego, CA) while power calculations used StatMate 2.00 (GraphPad Software Inc).

\footnotetext{
Acknowledgements

This work was supported by National Institutes of Health R01 MH62701, K23

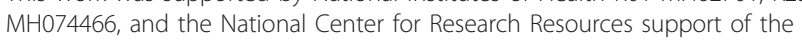
University of California San Francisco-Clinical and Translational Sciences Institute, UL1 RR024131. Its contents are solely the responsibility of the authors and do not represent the official views of the NIH. E.L.H. was a recipient of a Clinical Research Training Fellowship from the American Academy of Neurology.

These study results were presented in preliminary fashion at the Conference on Retroviruses and Opportunistic Infections (CROI) 2010 (Poster \#426) in San Francisco, February 2010.
}

\section{Author details}

'Department of Neurology' University of California San Francisco, San Francisco, CA, USA. ²Division of Biological Chemistry, Biocentre, Innsbruck Medical University, Innsbruck, Austria. ${ }^{3}$ Division of Experimental Medicine, Department of Medicine, University of California San Francisco, San Francisco, CA, USA. ${ }^{4}$ Department of Neurology, University of Washington, Seattle, WA, USA. 'Department of Neurology, Yale University, New Haven, CT, USA.

\section{Authors' contributions}

ELH examined study participants, performed lumbar punctures, and assisted with the analysis of the data and preparation of the manuscript. SSS examined study participants, performed lumbar punctures, and assisted in design of the study and reviewed the manuscript. EL served as the patient study coordinator, aided in the design of the study, performed the quantitative neurological performance testing and managed the data. DF performed assays of CSF and plasma neopterin. ES designed the flow cytometry assays, directed the SFGH Clinical Immunology Laboratory that performed the flow cytometry assays and CSF CCL2 ELISA assays, and analysed and interpreted flow cytometry data. RWP designed and oversaw the study, examined study participants, performed lumbar punctures, analysed and interpreted the data, and participated in preparation of the manuscript. All authors read and approved of the final manuscript.

\section{Competing interests}

Dr. Price has received funding from Merck to support an investigatorinitiated research study and an honorarium from Abbott for a conference presentation. The other authors have no competing interests.

Received: 14 January 2011 Accepted: 12 May 2011

Published: 12 May 2011

\section{References}

1. Davis LE, Hjelle BL, Miller VE, Palmer DL, Llewellyn AL, Merlin TL, Young SA, Mills RG, Wachsman W, Wiley CA: Early viral brain invasion in iatrogenic human immunodeficiency virus infection. Neurology 1992, 42(9):1736-1739.

2. Pilcher CD, Shugars DC, Fiscus SA, Miller WC, Menezes P, Giner J, Dean B, Robertson $\mathrm{K}$, Hart CE, Lennox JL, et al: HIV in body fluids during primary HIV infection: implications for pathogenesis, treatment and public health. AIDS 2001, 15(7):837-845.

3. Ellis RJ, Hsia K, Spector SA, Nelson JA, Heaton RK, Wallace MR, Abramson I, Atkinson JH, Grant I, McCutchan JA: Cerebrospinal fluid human immunodeficiency virus type 1 RNA levels are elevated in neurocognitively impaired individuals with acquired immunodeficiency syndrome. HIV Neurobehavioral Research Center Group. Ann Neurol 1997, 42(5):679-688.

4. McArthur JC, McClernon DR, Cronin MF, Nance-Sproson TE, Saah AJ, St Clair M, Lanier ER: Relationship between human immunodeficiency virusassociated dementia and viral load in cerebrospinal fluid and brain. Ann Neurol 1997, 42(5):689-698.

5. Spudich SS, Nilsson AC, Lollo ND, Liegler TJ, Petropoulos CJ, Deeks SG, Paxinos EE, Price RW: Cerebrospinal fluid HIV infection and pleocytosis: relation to systemic infection and antiretroviral treatment. BMC Infect Dis 2005, 5:98.

6. Spudich S, Lollo N, Liegler T, Deeks SG, Price RW: Treatment benefit on cerebrospinal fluid HIV-1 levels in the setting of systemic virological suppression and failure. J Infect Dis 2006, 194(12):1686-1696.

7. Gisslen M, Fuchs D, Svennerholm B, Hagberg L: Cerebrospinal fluid viral load, intrathecal immunoactivation, and cerebrospinal fluid monocytic cell count in HIV-1 infection. J Acquir Immune Defic Syndr 1999, 21(4):271-276.

8. Abdulle S, Hagberg L, Svennerholm B, Fuchs D, Gisslen M: Continuing intrathecal immunoactivation despite two years of effective antiretroviral therapy against HIV-1 infection. AIDS 2002, 16(16):2145-2149.

9. Cinque P, Brew BJ, Gisslen M, Hagberg L, Price RW: Cerebrospinal fluid markers in central nervous system HIV infection and AIDS dementia complex. Handb Clin Neurol 2007, 85:261-300.

10. Sinclair $E$, Ronquillo R, Lollo N, Deeks SG, Hunt P, Yiannoutsos CT, Spudich S, Price RW: Antiretroviral treatment effect on immune activation reduces cerebrospinal fluid HIV-1 infection. J Acquir Immune Defic Syndr 2008, 47(5):544-552.

11. Hagberg L, Cinque P, Gisslen M, Brew BJ, Spudich S, Bestetti A, Price RW, Fuchs D: Cerebrospinal fluid neopterin: an informative biomarker of central nervous system immune activation in HIV-1 infection. AIDS Res Ther 2010, 7:15.

12. Navia BA, Jordan BD, Price RW: The AIDS dementia complex: I. Clinical features. Ann Neurol 1986, 19(6):517-524.

13. Antinori A, Arendt G, Becker JT, Brew BJ, Byrd DA, Cherner M, Clifford DB, Cinque P, Epstein LG, Goodkin K, et al: Updated research nosology for 
HIV-associated neurocognitive disorders. Neurology 2007, 69(18):1789-1799.

14. Kaul M, Zheng J, Okamoto S, Gendelman HE, Lipton SA: HIV-1 infection and AIDS: consequences for the central nervous system. Cell Death Differ 2005, 12(Suppl 1):878-892.

15. Gonzalez-Scarano F, Martin-Garcia J: The neuropathogenesis of AIDS. Nat Rev Immunol 2005, 5(1):69-81

16. Simioni S, Cavassini M, Annoni JM, Rimbault Abraham A, Bourquin I, Schiffer V, Calmy A, Chave JP, Giacobini E, Hirschel B, et al: Cognitive dysfunction in HIV patients despite long-standing suppression of viremia. AIDS 2010, 24(9):1243-1250.

17. Heaton RK, Clifford DB, Franklin DR Jr, Woods SP, Ake C, Vaida F, Ellis RJ, Letendre SL, Marcotte TD, Atkinson JH, et al: HIV-associated neurocognitive disorders persist in the era of potent antiretroviral therapy: CHARTER Study. Neurology 2010, 75(23):2087-2096.

18. Tozzi V, Balestra P, Bellagamba R, Corpolongo A, Salvatori MF, ViscoComandini U, Vlassi C, Giulianelli M, Galgani S, Antinori A, et al: Persistence of neuropsychologic deficits despite long-term highly active antiretroviral therapy in patients with HIV-related neurocognitive impairment: prevalence and risk factors. J Acquir Immune Defic Syndr 2007, 45(2):174-182.

19. Eden A, Price RW, Spudich S, Fuchs D, Hagberg L, Gisslen M: Immune activation of the central nervous system is still present after $>4$ years of effective highly active antiretroviral therapy. J Infect Dis 2007, 196(12):1779-1783.

20. Ancuta P, Kamat A, Kunstman KJ, Kim EY, Autissier P, Wurcel A, Zaman T, Stone $\mathrm{D}$, Mefford M, Morgello $\mathrm{S}$, et al: Microbial translocation is associated with increased monocyte activation and dementia in AIDS patients. PLOS One 2008, 3(6):e2516.

21. Uthman OA, Abdulmalik JO: Adjunctive therapies for AIDS dementia complex. Cochrane Database Syst Rev 2008, , 3: CD006496.

22. Si Q, Cosenza M, Kim MO, Zhao ML, Brownlee M, Goldstein H, Lee S: A novel action of minocycline: inhibition of human immunodeficiency virus type 1 infection in microglia. J Neurovirol 2004, 10(5):284-292.

23. Zink MC, Uhrlaub J, DeWitt J, Voelker T, Bullock B, Mankowski J, Tarwater P, Clements J, Barber S: Neuroprotective and anti-human immunodeficiency virus activity of minocycline. JAMA 2005, 293(16):2003-2011.

24. Follstaedt SC, Barber SA, Zink MC: Mechanisms of minocycline-induced suppression of simian immunodeficiency virus encephalitis: inhibition of apoptosis signal-regulating kinase 1. J Neurovirol 2008, 14(5):376-388

25. Clements JE, Mankowski JL, Gama L, Zink MC: The accelerated simian immunodeficiency virus macaque model of human immunodeficiency virus-associated neurological disease: from mechanism to treatment. J Neurovirol 2008, 14(4):309-317.

26. Ratai EM, Bombardier JP, Joo CG, Annamalai L, Burdo TH, Campbell J, Fell R, Hakimelahi R, He J, Autissier P, et al: Proton magnetic resonance spectroscopy reveals neuroprotection by oral minocycline in a nonhuman primate model of accelerated NeuroAIDS. PLoS One 2010, 5(5):e10523.

27. Szeto GL, Brice AK, Yang HC, Barber SA, Siliciano RF, Clements JE: Minocycline attenuates HIV infection and reactivation by suppressing cellular activation in human CD4+ T cells. J Infect Dis 2010, 201(8):1132-1140.

28. Chen M, Ona VO, Li M, Ferrante RJ, Fink KB, Zhu S, Bian J, Guo L, Farrell LA, Hersch SM, et al: Minocycline inhibits caspase-1 and caspase-3 expression and delays mortality in a transgenic mouse model of Huntington disease. Nat Med 2000, 6(7):797-801.

29. Lin S, Zhang Y, Dodel R, Farlow MR, Paul SM, Du Y: Minocycline blocks nitric oxide-induced neurotoxicity by inhibition p38 MAP kinase in rat cerebellar granule neurons. Neurosci Lett 2001, 315(1-2):61-64.

30. Du Y, Ma Z, Lin S, Dodel RC, Gao F, Bales KR, Triarhou LC, Chernet E, Perry KW, Nelson DL, et al: Minocycline prevents nigrostriatal dopaminergic neurodegeneration in the MPTP model of Parkinson's disease. Proc Natl Acad Sci USA 2001, 98(25):14669-14674.

31. Van Den Bosch L, Tilkin P, Lemmens G, Robberecht W: Minocycline delays disease onset and mortality in a transgenic model of ALS. Neuroreport 2002, 13(8):1067-1070.

32. Wu DC, Jackson-Lewis V, Vila M, Tieu K, Teismann P, Vadseth C, Choi DK, Ischiropoulos $\mathrm{H}$, Przedborski S: Blockade of microglial activation is neuroprotective in the 1-methyl-4-phenyl-1,2,3,6-tetrahydropyridine mouse model of Parkinson disease. J Neurosci 2002, 22(5):1763-1771.
33. Metz $L M$, Zhang $Y$, Yeung $M$, Patry DG, Bell RB, Stoian CA, Yong WW, Patten SB, Duquette P, Antel JP, et al: Minocycline reduces gadoliniumenhancing magnetic resonance imaging lesions in multiple sclerosis. Ann Neurol 2004, 55(5):756.

34. Michel-Monigadon D, Nerriere-Daguin V, Leveque X, Plat M, Venturi $E_{\text {, }}$ Brachet $P$, Naveilhan $P$, Neveu I: Minocycline promotes long-term survival of neuronal transplant in the brain by inhibiting late microglial activation and T-cell recruitment. Transplantation 2010, 89(7):816-823.

35. Guimaraes JS, Freire MA, Lima RR, Picanco-Diniz CW, Pereira A, GomesLeal W: Minocycline treatment reduces white matter damage after excitotoxic striatal injury. Brain Res 2010, 1329:182-193.

36. Price RW, Staprans S: Measuring the "viral load" in cerebrospinal fluid in human immunodeficiency virus infection: window into brain infection? Ann Neurol 1997, 42(5):675-678.

37. Price RW: The two faces of HIV infection of cerebrospinal fluid. Trends Microbiol 2000, 8(9):387-391.

38. Wirleitner B, Schroecksnadel K, Winkler C, Fuchs D: Neopterin in HIV-1 infection. Mol Immunol 2005, 42(2):183-194.

39. Zink MC, Clements JE: A novel simian immunodeficiency virus model that provides insight into mechanisms of human immunodeficiency virus central nervous system disease. J Neurovirol 2002, 8(Suppl 2):42-48.

40. Mankowski JL, Queen SE, Clements JE, Zink MC: Cerebrospinal fluid markers that predict SIV CNS disease. J Neuroimmunol 2004, 157(12):66-70.

41. Shacklett BL, Cox CA, Wilkens DT, Karl Karlsson R, Nilsson A, Nixon DF, Price RW: Increased adhesion molecule and chemokine receptor expression on CD8+ T cells trafficking to cerebrospinal fluid in HIV-1 infection. J Infect Dis 2004, 189(12):2202-2212.

42. Neuenburg JK, Cho TA, Nilsson A, Bredt BM, Hebert SJ, Grant RM, Price RW: T-cell activation and memory phenotypes in cerebrospinal fluid during HIV infection. J Acquir Immune Defic Syndr 2005, 39(1):16-22.

43. Tikka T, Fiebich BL, Goldsteins G, Keinanen R, Koistinaho J: Minocycline, a tetracycline derivative, is neuroprotective against excitotoxicity by inhibiting activation and proliferation of microglia. J Neurosci 2001, 21(8):2580-2588.

44. Tikka TM, Koistinaho JE: Minocycline provides neuroprotection against $\mathrm{N}$ methyl-D-aspartate neurotoxicity by inhibiting microglia. J Immunol 2001, 166(12):7527-7533.

45. Kloppenburg M, Verweij CL, Miltenburg AM, Verhoeven AJ, Daha MR, Dijkmans BA, Breedveld FC: The influence of tetracyclines on T cell activation. Clin Exp Immunol 1995, 102(3):635-641.

46. Maier K, Merkler D, Gerber J, Taheri N, Kuhnert AV, Williams SK, Neusch C, Bahr M, Diem R: Multiple neuroprotective mechanisms of minocycline in autoimmune CNS inflammation. Neurobiol Dis 2007, 25(3):514-525.

47. Yrjanheikki J, Tikka T, Keinanen R, Goldsteins G, Chan PH, Koistinaho J: A tetracycline derivative, minocycline, reduces inflammation and protects against focal cerebral ischemia with a wide therapeutic window. Proc Natl Acad Sci USA 1999, 96(23):13496-13500.

48. Hersch S, Fink K, Vonsattel JP, Friedlander RM: Minocycline is protective in a mouse model of Huntington's disease. Ann Neurol 2003, 54(6):841, author reply 842-843.

49. Hunter CL, Bachman D, Granholm AC: Minocycline prevents cholinergic loss in a mouse model of Down's syndrome. Ann Neurol 2004, 56(5):675-688.

50. Stirling DP, Khodarahmi K, Liu J, McPhail LT, McBride CB, Steeves JD, Ramer MS, Tetzlaff W: Minocycline treatment reduces delayed oligodendrocyte death, attenuates axonal dieback, and improves functional outcome after spinal cord injury. J Neurosci 2004, 24(9):2182-2190

51. Ryu JK, Franciosi S, Sattayaprasert P, Kim SU, McLarnon JG: Minocycline inhibits neuronal death and glial activation induced by beta-amyloid peptide in rat hippocampus. Glia 2004, 48(1):85-90.

52. Kim HS, Suh YH: Minocycline and neurodegenerative diseases. Behav Brain Res 2009, 196(2):168-179.

53. Tilley BC, Alarcon GS, Heyse SP, Trentham DE, Neuner R, Kaplan DA, Clegg DO, Leisen JC, Buckley L, Cooper SM, et al: Minocycline in rheumatoid arthritis. A 48-week, double-blind, placebo-controlled trial. MIRA Trial Group. Ann Intern Med 1995, 122(2):81-89.

54. Lampl Y, Boaz M, Gilad R, Lorberboym M, Dabby R, Rapoport A, AncaHershkowitz M, Sadeh M: Minocycline treatment in acute stroke: an open-label, evaluator-blinded study. Neurology 2007, 69(14):1404-1410. 
55. Zabad RK, Metz LM, Todoruk TR, Zhang Y, Mitchell JR, Yeung M, Patry DG, Bell $\mathrm{RB}$, Yong $\mathrm{W}$ : The clinical response to minocycline in multiple sclerosis is accompanied by beneficial immune changes: a pilot study. Mult Scler 2007, 13(4):517-526.

56. Yilmaz A, Price RW, Spudich S, Fuchs D, Hagberg L, Gisslen M: Persistent intrathecal immune activation in HIV-1-infected individuals on antiretroviral therapy. J Acquir Immune Defic Syndr 2008, 47(2):168-173.

57. Mildvan D, Spritzler J, Grossberg SE, Fahey JL, Johnston DM, Schock BR, Kagan J: Serum neopterin, an immune activation marker, independently predicts disease progression in advanced HIV-1 infection. Clin Infect Dis 2005, 40(6):853-858.

58. Cinque $P$, Vago L, Mengozzi M, Torri V, Ceresa D, Vicenzi E, Transidico P, Vagani A, Sozzani S, Mantovani A, et al: Elevated cerebrospinal fluid levels of monocyte chemotactic protein-1 correlate with HIV-1 encephalitis and local viral replication. AIDS 1998, 12(11):1327-1332.

59. Neuenburg JK, Furlan S, Bacchetti P, Price RW, Grant RM: Enrichment of activated monocytes in cerebrospinal fluid during antiretroviral therapy. AIDS 2005, 19(13):1351-1359.

60. Deeks SG, Kitchen CM, Liu L, Guo H, Gascon R, Narvaez AB, Hunt P, Martin JN, Kahn JO, Levy J, et al: Immune activation set point during early HIV infection predicts subsequent CD4+ T-cell changes independent of viral load. Blood 2004, 104(4):942-947.

61. Hunt PW: Role of immune activation in HIV pathogenesis. Curr HIV/AIDS Rep 2007, 4(1):42-47.

62. Hunt PW, Brenchley J, Sinclair E, McCune JM, Roland M, Page-Shafer K, Hsue P, Emu B, Krone M, Lampiris H, et al: Relationship between T cell activation and CD4+ T cell count in HIV-seropositive individuals with undetectable plasma HIV RNA levels in the absence of therapy. I Infect Dis 2008, 197(1):126-133.

63. Harrington PR, Haas DW, Ritola K, Swanstrom R: Compartmentalized human immunodeficiency virus type 1 present in cerebrospinal fluid is produced by short-lived cells. J Virol 2005, 79(13):7959-7966.

64. Ritola K, Robertson K, Fiscus SA, Hall C, Swanstrom R: Increased human immunodeficiency virus type 1 (HIV-1) env compartmentalization in the presence of HIV-1-associated dementia. J Virol 2005, 79(16):10830-10834.

65. Harrington PR, Schnell G, Letendre SL, Ritola K, Robertson K, Hall C, Burch CL, Jabara CB, Moore DT, Ellis RJ, et al: Cross-sectional characterization of HIV-1 env compartmentalization in cerebrospinal fluid over the full disease course. AIDS 2009, 23(8):907-915.

66. Schnell G, Spudich S, Harrington P, Price RW, Swanstrom R: Compartmentalized human immunodeficiency virus type 1 originates from long-lived cells in some subjects with HIV-1-associated dementia. PLoS Pathog 2009, 5(4):e1000395.

67. Schnell G, Price RW, Swanstrom R, Spudich S: Compartmentalization and clonal amplification of HIV-1 variants in the cerebrospinal fluid during primary infection. J Virol 2010, 84(5):2395-2407.

68. Price RW, Brew BJ: The AIDS dementia complex. J Infect Dis 1988, 158(5):1079-1083.

69. Probasco JC, Spudich SS, Critchfield J, Lee E, Lollo N, Deeks SG, Price RW: Failure of atorvastatin to modulate CSF HIV-1 infection: results of a pilot study. Neurology 2008, 71(7):521-524.

70. Link H, Tibbling G: Principles of albumin and IgG analyses in neurological disorders. III. Evaluation of IgG synthesis within the central nervous system in multiple sclerosis. Scand J Clin Lab Invest 1977, 37(5):397-401.

71. Tibbling G, Link H, Ohman S: Principles of albumin and IgG analyses in neurological disorders. I. Establishment of reference values. Scand J Clin Lab Invest 1977, 37(5):385-390.

72. Price RW, Yiannoutsos CT, Clifford DB, Zaborski L, Tselis A, Sidtis JJ, Cohen B, Hall CD, Erice A, Henry K: Neurological outcomes in late HIV infection: adverse impact of neurological impairment on survival and protective effect of antiviral therapy. AIDS Clinical Trial Group and Neurological AIDS Research Consortium study team. AIDS 1999, 13(13):1677-1685.

doi:10.1186/1742-6405-8-17

Cite this article as: Ho et al:: Minocycline fails to modulate cerebrospinal fluid HIV infection or immune activation in chronic untreated HIV-1 infection: results of a pilot study. AIDS Research and Therapy 2011 8:17.

\section{Submit your next manuscript to BioMed Central and take full advantage of:}

- Convenient online submission

- Thorough peer review

- No space constraints or color figure charges

- Immediate publication on acceptance

- Inclusion in PubMed, CAS, Scopus and Google Scholar

- Research which is freely available for redistribution 